\title{
ESTIMATION OF THE VALUE OF TRAVEL TIME: MODELS CONSIDERING BINARY AND MULTI MODE CHOICE
}

\author{
John Kraft and Arthur Kraft** \\ University of Florida and Ohio University
}

\section{INTRODUCTION}

Recent empirical investigations into the mode choice decisions of travelers has resulted in the estimation of the value which individuals placed on time spent in travel. In most cases the individual's choice among competing travel modes is based on the cost and the time characteristics of the modes under consideration. The individual's value of travel time is then allowed to influence his mode choice decision.

Many of the past mode choice models contained two deficiences. First, the time and cost characteristics of the modes did not reflect the complete cost and time associated with the journey, but consideredonly the ticket cost and the time spent on the particular mode. This resulted in an error in the estimation of the value of travel time. It can be corrected by considering costs and times from origin to destination rather than terminal to terminal. The total time paramete $r$ is then the linear combination of time spent on the travel mode, access time and egress time, and the average waiting time at the origin for the scheduled arrival of the mode. Second, in the past most of the mode choice models computed the value placed ontravel time in a binary choice situation. This was particularly true of intra-urban travel models where the individual's choice is between his own automobile and some form of mass transit. The probability choice was then binary, in that the individual either did or did not drive his own automobile. This mode choice decision is quite unrealistic in an inter-urban travel model since the choice is usually between more than two modes (rail, auto, bus or airplane).

\section{REVIEW OF MODE CHOICE MODELS}

The Moses and Williamson [11] approach and the Beesley[2] approach are primarilytrade-off methods. Their usefulness is somewhat of a limited nature in that both are restricted to a binary comparison. Moses and Williamson focus on atrade-off between income and leisure as sociated with the use of a particular mode and then take the difference in net income in a comparison of two transport modes. For any particular mode $k$ the individual has a net income where $\mathrm{L}$ is given level of leisure, $\mathrm{s}$ is worker's stock of time, w is

$$
\mathrm{Y}_{\mathrm{k}}=\mathrm{w}\left(\mathrm{s}-\mathrm{L}-\mathrm{t}_{\mathrm{k}}\right)-\mathrm{c}_{\mathrm{k}}
$$

wage rate per unit of time, $c_{k}$ is money cost of mode $k$, and $t_{k}$ is time spent on mode $k$. The comparison between mode $k$ and an alternative gives

$$
\mathrm{z}=\mathrm{Y}_{\mathrm{k}}-\mathrm{Y}_{1}=\mathrm{w}\left(\mathrm{t}_{1}-\mathrm{t}_{\mathrm{k}}\right)+\mathrm{c}_{1}-\mathrm{c}_{\mathrm{k}} \text {. }
$$

If $z>0$ the individual prefers mode $k$, if $z<0$ he prefers the alternative, and if $z=0$ he is indifferent. Beesley presented the modal choice as an inequality such that for the preferred mode the sum of its travel time (a) and its cost (b) is less than similar quantities ( $x$ and $y$ respectively) for an inferior mode. 
If, however, the performance of the preferred mode is not superior in time and cost, the choice mechanism and the form of the time-- cost trade-off may be obscured. Unless absolute superiority on all characteristics exists, a choice could not be made.

Reuben Gronau [5] developed a model to measure the demand for a particular mode as well as the value of time for this mode. It is used to test Gary Becker's theory [1] on the allocation of time. The quantity of trips demanded $(x)$ is a linea $r$ function of their price and the passenger's income,

$$
\mathbf{x}=\alpha_{0}+\beta_{1} \Pi+\beta_{2} y+u
$$

where $I$ is price of travel, $y$ is individual's income, and $u=$ stochastic disturbance term. Gronau feels that the price variable has both a time and money component.

$$
\mathbf{x}=\alpha_{0}+\beta_{1}(P+K T)+\beta_{2}^{\gamma}+u
$$

where $\mathrm{P}$ is the money component and $\mathrm{KT}$ the time component with $\mathrm{K}$ being the value of time. Equation 4 becomes

$$
\mathbf{x}=\alpha_{0}+\beta_{1} P+\gamma_{1} T+\beta_{2} Y+u
$$

The multiple regression of the number of trips on the money outlays, elapsed time, and income would yield estimates of the price effect $\left(\beta_{1}\right)$, income effect $\left(\beta_{2}\right)$ and the price or value of time $\left(K=\gamma_{1} / \beta_{1}\right)$.

The final conclusion of the study is that the price of time is about forty per cent of the travelers' average hourly wage rate.

The trade-off ratio concept was developed by Rodney Plourde [13]. The trade-off rate expresses how a small change in value of one travel attribute will affect a correspondingly small change in the value. of a nother travel attribute at some constant travel volume. In specific, the trade-off ratio for mode $\mathrm{k}$ would represent a trade-off between the cost and time characteristics of the mode. The general equation in linear form is

$$
\mathrm{Y}_{\mathrm{k}}=\beta_{0}+\beta_{\mathrm{T}} \mathrm{X}_{\mathrm{T}}+\beta_{\mathrm{c}} \mathrm{X}_{\mathrm{c}}
$$

where $\mathrm{Y}_{\mathrm{k}}$ is number of trips by passengers from i to $\mathrm{j}$ by mode $\mathrm{k}$ (travel volumes), $\mathrm{X}_{\mathrm{T}}$ is travel time by mode $\mathrm{k}$ between $\mathrm{i}$ and $\mathrm{j}$, and $\mathrm{X}_{\mathrm{C}}$ is cost by mode $\mathrm{k}$ between $\mathrm{i}$ and $\mathrm{j}$.

Take the derivative of travel volume with respect to the two attributes to determine how much "a small change in the value of one attribute will af fect a correspondingly small change in the value of a nother service attribute such that a constant travel volume will be maintained. "[13].

The effect of a small change in time on a small change in cost is given by

$$
\mathrm{T}_{\mathrm{T} c}=\frac{\frac{\mathrm{dY}}{\mathrm{dX}}}{\frac{\mathrm{dY}}{\mathrm{dX}}}=\frac{\beta_{\mathrm{T}}}{\beta_{\mathrm{c}}}=\frac{\frac{\text { trips }}{\text { minutes }}}{\frac{\text { trips }}{\text { cents }}}=\frac{\text { cents }}{\text { minutes }}
$$

Plourde obtained values of time spent intravel which were $\$ 3.48$ per hour for the best mode and $\$ 2.36$ per hour for other modes. 
A last measure of the value of time is given in the work of Thomas Lisco [10]. His work is also a study of the marginal value of travel time and its influence on consumer choice. Again, this is a time value study in binary choice. A cumulative normal curve is fitted to the data through probit analysis. Multiple probit analysis assumes that the relation between the probability of a given result and the vector of the independent variable has the form

$$
P(x)=f(Y)=\frac{1}{2} \int_{-\infty}^{\gamma} e^{-t^{2} / 2} d t
$$

where $(P(x))$ is the probability that a given mode is chosen. $Y$ is a linear function of the vector of independent variables values and $t$ is $(\mu-y)$ where $\mu$ is normally distributed with mean Y and variance one. The final estimating equation is the linear probit equation

$$
\mathrm{Y}=\beta_{0}+\beta_{1} \Delta \mathrm{T}+\beta_{2} \Delta \mathrm{M}+\sum_{\mathrm{i}=1}^{\mathrm{k}} \beta_{\mathrm{i}} \mathrm{X}_{\mathrm{i}}
$$

where $\mathrm{Y}$ is the probability of traveling by automobile expressed in "probits" (unit cumulative normal transform of $P(x)$ ), $\Delta T$ is transit time minus driving time, $\Delta \mathrm{M}$ is driving costminustransit cost, and $\mathrm{X}_{\mathrm{i}}$ is other travel characteristics.

The major difference between the probit equation and the standard linear regression is that the dependentvariable is not a linearfunction of $Y$, but its unit cumulative normal transform $P(x)$. The $\beta^{\prime} s$ a re not estimated but obtained by iteration. 1 In the probit equation the implied value of time is obtained by the ratio $\beta_{1} / \beta_{2}$. After manipulation Lisco obtained a value of $\$ 2.52$ per hour as the value placed on time spent in travel.

\section{MULTI-MODE CHOICE MODEL}

The data collected is cross sectional data of thirty-two city pairs in the northeast corridor of the United States for the year 1961. Each of the thirtytwo city pairs is considered to be a separteobservation for each mode. This is acceptable since the abstract mode approach assumes that the consumer choice among modes between any city pair is independent of the size of the city pairs and their distance apart. ${ }^{2}$

The data collected were on the attributes of railroad, air and bus for thirty-two city pairs. The travel volume data was on one-way passenger trips between city pairs by the modes considered in the study. The passenger volume data was collected on a terminal to terminal to terminal basis rather than actual city to city. Thus an individual traveling may be passing through a terminal without it being either the origin or destination of his trip.

Journey times, money costs, and frequencies of departure were computed from actual schedules. Money costs of travel on any mode between city pairs were the sum of access cost to the terminal from the center city, the ticket cost of the mode, and egress cost from the terminal to the center city. Journey times were obtained in a similar manner being the sum of time required for access to the terminal, time spent on the mode, and egress time from the terminal. The daily frequencies of departure by anymode between cities were taken from schedules. Where there were two or more terminals for a mode, that is two airports for New York City, etcetera, then times and costs were averaged for the two facilities and frequencies of departure were added. The city pair data was collected formetropolitan populations capable of supporting the travel volumes given. The construction and collection of the data was done from time schedules and surveys undertaken by the Depart- 
ment of Commerce for the Northeast Corridor Transportation Project.

The data contains time parameters on access time, trip time, and egress time; however, there are no values on average waiting time, which is considered to be a part of the total travel time. If an individual possesses his own private means of transportation the average waiting time is in fact zero or infinitesimal. Commercial modes of transportation do not have this characteristic since the traveler does not control the mode. Individuals are limited to the scheduled arrivals and departures of the mode and their own random arrival may not coincide with the departure of the mode. From the other point of view, individuals will not necessarily arrive in a completely random manner, but may arrange to arrive so as to minimize their waiting time prior to the scheduled departure time as listed. It is also generally acknowledged that modes do not always leave and depart in accordance with printed schedules and this results in a situation that fairly well approximates a random arrival time in terms of waiting time generated. The variable to be converted to waiting time is frequency of departure of mode $k$ for city pair $\mathrm{i}$ and $\mathrm{j}[6]$.

We will now discuss the methods of estimating the value of time. In the first approach an implied user value of time is computed. The time and cost parameters are available for all modes on each arc of the network. The cost parameter includes the entire travel cost and not just the ticket cost. The time parameter reflects the total travel time and is constructed from access time, waiting time, trip time, and egress time. For any mode $\mathrm{k}$ the time parameters and the money outlay (cost) parameters a re linear functions of the distance traveled. Since the distance is the same, and time and cost parameters may be expressed in terms of each other. For any mode $k$ the mean ratio of the cost and time parameters over the $m$ arcs of the network results in an implied user value of time which is given as follows:

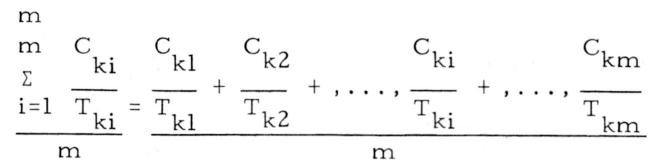

where $\mathrm{C}_{\mathrm{ki}}$ is the cost outlay for mode $\mathrm{k}$ on arc $\mathrm{i}, \mathrm{T}_{\mathrm{ki}}$ is the time elapsed for mode $k$ on arc $i$, and $m$ is the number of arcs in the network.

This is somewhat similar to a trade-off ratio where an average value of time is obtained. Since both cost and time are linear functions of distance, any change in cost must be reflected in a change in the time of the journey by mode k. On the basis of the above expression the value of time is computed as a value which users place on the time spent intravel on mode $k$. For our data, the value of time is computed for each mode in cents per minute and converted to dollars per hour. The values appear in Table 2 .

Table 2. Computed User Values for Time Spent in Travel

Mode

Rail

Air

Bus
Value of Time

(cents/minute)
Value of Time (dollars / hour) 
The values computed by this method compare favorably with values of time by mode which were given in the studies reviewed in Section II. The mean user value of time was highest for the more costly and faster mode, where a premium is placed on reducing time spent in travel. Those choosing the bus will place a lower priority on time, than airplane users. Yet, it must be pointed out that these values a re not as widely spread as they could be if the distances considered were of a greater magnitude. The longest distance is the Boston to Washington city pair which is approximately 437 miles. For the other thirty-one city pairs the distance is considerably less than 250 miles.

The second model to be presented is a probability model of modal choice in terms of cost and time ratios of the mode. Let us assume we are working with two modes. The cost and time ratios are available for modes 1 and 2 . Let $\mathrm{p}$ be the probability of choosing mode 1 and the 1 - $p$ is the probability of selecting mode 2 . The linear specification is

$$
\mathrm{p}=\mathrm{a}+\mathrm{b} \frac{\mathrm{T}_{1}}{\mathrm{~T}_{2}}+\mathrm{c} \frac{\mathrm{C}_{1}}{\mathrm{C}_{2}}
$$

where $T_{1}$ and $T_{2}$ are the times for two competing modes and $C_{1}$ and $C_{2}$ are their costs. This linear probability function model has a basic fault in that because the relationship is linear it is possible for larger values of the time and cost ratios to give probabilities greater than one. The problem could be remedied by using the probit transform or we could take the log of the probability ratios. In logarithmic form of the probability ratio becomes

$$
\log \frac{p}{1-p}=a+b \log \frac{T_{1}}{T_{2}}+c \log \frac{C_{1}}{C_{2}} .
$$

The left side variable in 12 is known as the logit and corresponds to the probability that mode 1 is selected. The logit is a monotonically increasing function varying between $-\infty$ and $+\infty$. Thus the logit transform of the probability is not confined to the finite interval $(0,1)$ as is $p$. This is quite convenient since the right side of equation 12 canusually take any a rbitrary real values.

The logit owes its name to the logistic curve to which it has a relation similar to that of the probit to the normal curve. In such cases as equation 12 where the logit of the probabilities is between an event and its complement, the method of estimating the relationship is to compute a logit regression which is based on the model

$$
\log \frac{\mathrm{p}_{1}}{1-\mathrm{p}_{1}}=\alpha+\beta_{1} \log \frac{\mathrm{T}_{1}}{\mathrm{~T}_{2}}+\beta_{2} \log \frac{\mathrm{C}_{1}}{\mathrm{C}_{2}}
$$

where $\alpha, \beta_{1}$ and $\beta_{2}$ are parameters to be estimated [16, p. 71]. In a binary choice situation we may use either the probit or the logit transform. However, if the decision is among three modes, the non binary nature of this choice decision rules out the use of probit analysis. The multinomialextension of the linear model 12 makes it possible to compare more than two alternatives. Henri Theil [17] has proved an extension of the linear logit model. This effort is based on Theil's multinomial extension of the logit function and will be used to compute the value of travel time.

Assume the re are $\mathrm{N}$ alternatives with probabilities $\mathrm{p}_{1}, \mathrm{p}_{2}, \ldots, \mathrm{p}_{\mathrm{N}}$ On any arc of the network one of the alternatives must be realized, and hence 
The extension of 12 to $\mathrm{N}$ probabilities is

$$
\frac{P_{i}}{P_{j}}=e^{\alpha}{ }^{\alpha j} \prod_{k=1}^{n}\left(\frac{y_{k i}}{y_{k i}}\right)^{\gamma_{k i j}} \quad i, j=1, \ldots, N
$$

The variables in the ratio $_{3}\left(y_{k i}, k=1, \ldots, n, i=1, \ldots, N\right)$ correspond to the time and cost ratios of 12 . $^{3}$

Interchanging the indices $(i, j)$ is equivalent to taking the reciprocal of log transform of the left side of

$$
\log \frac{p_{i}}{p_{j}}=-\log \frac{p_{j}}{p_{i}} \text {. }
$$
$\mathrm{k}$.

Thus the relations $\alpha_{i j}=-\alpha_{j i}$ and $\gamma_{k i j}=\gamma_{k j i}$ hold for all values of $i, j$ and

Since the choice of indices of $\mathrm{i}$ and $\mathrm{j}$ is arbitrary, ${ }^{\alpha}{ }_{\mathrm{i}} \mathrm{i}$ is of the form $\left(\alpha_{i}-\alpha_{j}\right)$ and $\gamma_{k i j}$ is independent of the last two subscripts. "The multinomial

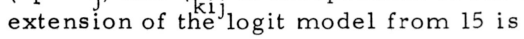

$$
\frac{P_{i}}{P_{j}}=e^{\alpha_{i}-\alpha_{j}} \prod_{k=1}^{n}\left(\frac{y_{k i}}{y_{k i}}\right)^{\gamma k} \quad \begin{array}{rlll}
i, j & =1, \ldots, N \\
k & =1, \ldots, n .
\end{array}
$$

The variables which are in the ratios ( $\left.y^{\prime} s\right)$ will have exponents ( $\gamma^{\prime} s$ ) which will be the same regardless of the probability ratios on the left side, that is the exponents are independent of $\mathrm{i}$ and $\mathrm{j}[17, \mathrm{p} .253$ ].

We will now turn to the specific form of our logit model and the computation of the value of time. In our model three transport modes such that $\mathrm{N}=3$ and each mode has two characteristics $n=2.4$ On any arc of the network one has a choice among three modes where the three alternatives have probabilities $\mathrm{p}_{1}, \mathrm{p}_{2}$, and $\mathrm{p}_{3}$ which sum to one. Next extend to the case of three probabilities where $i, j=1,2,3$. Multiply the corresponding sides of equation 15 by $(i, j)=(1,2)$ and $(i, j)=(2,3)$. That yields

$$
\frac{p_{1}}{p_{2}} \cdot \frac{p_{2}}{p_{3}}=e^{\alpha 12+\alpha_{23}} \prod_{k=1}^{2}\left(\frac{y_{k 1}}{y_{k 2}}\right)^{\gamma_{k 12}}\left(\frac{y_{k 2}}{y_{k 3}}\right)^{\gamma_{k 23}}
$$

The left side of 18 becomes the ratio of $\mathrm{p}_{1}$ to $\mathrm{p}_{3}$,

$$
\frac{p_{1}}{p_{3}}=e^{\alpha_{13}} \underset{k=1}{n}\left(\frac{y_{k 1}}{y_{k 2}}\right)^{\gamma k 13}
$$

This implies that in comparing the right side of 18 and 19 the following conclusions a re apparent:

$$
\alpha_{12}+\alpha_{23}=\alpha_{13}
$$


The choice of indices $(i, j)=1,2,3$ is a rbitrary. This is seen by checking the relationship in 15 where

$$
e^{\alpha} i j=\frac{e^{\alpha i}}{e^{\alpha}}
$$

Then the choice of indices becomes

$$
\begin{aligned}
& \alpha_{12}=\alpha_{1}-\alpha_{2} \\
& \alpha_{23}=\alpha_{2}-\alpha_{3}
\end{aligned}
$$

and

$$
\alpha_{12}+\alpha_{23}=\left(\alpha_{1}-\alpha_{2}\right)+\left(\alpha_{2}-\alpha_{3}\right)=\alpha_{1}-\alpha_{3}
$$

where

$$
\alpha_{13}=\alpha_{1}-\alpha_{3}
$$

and

$$
\alpha_{12}+\alpha_{23}=\alpha_{13}
$$

for any arbitrary i, j, here i, j, = 1, 2, 3 for the three travel modes.

In our special case where $\mathrm{N}$ is equal to the number of modes (rail, air, bus) and $\mathrm{n}$ is the number of characteristics (time and cost), specification 18 becomes

$$
\frac{\mathrm{p}_{1}}{\mathrm{p}_{3}}=\mathrm{e}^{\alpha_{1}-3}\left(\frac{\mathrm{T}_{1}}{\mathrm{~T}_{3}}\right)^{\gamma 1}\left(\frac{\mathrm{C}_{1}}{\mathrm{C}_{3}}\right)^{\gamma 2}
$$

The variables in the ratios have exponents which are the same for a given characteristic whatever the probability ratios maybe. This independence of $i$ and $j$ is all that is needed to compute the value a traveler places on travel time. In this model the value of time is not mode specific, but rather a value the consumer places on travel time without regard to mode. Note that the expression 25 is sufficient for determining the value of time for three modes even though it only has the probability ratios for two modes. This determination is sufficient since their values are determined by the requirement that the probabilities sum to one.

The statistical estimation of 25 uses weighted least squares. The probabilities of the modes on any arc of the network a re computed as each mode's share of total travel volume on the arc. The probabilities of any a rc sum to one. The next step is to take the natural logarithmic transform of the probability ratio. The $\log$ of the probability ratio is the logit and is an increasing function of the probability ratio. The situation is one of a "logit regression." After statistical estimation of this logit equation

$$
\mathrm{Y}=\log \frac{\mathrm{p}_{1}}{\mathrm{p}_{3}}=\alpha_{13}+\gamma_{1} \log \frac{\mathrm{T}_{1}}{\mathrm{~T}_{3}}+\gamma_{2} \log \frac{\mathrm{C}_{1}}{\mathrm{C}_{3}}
$$

the value of time may be computed. The value of time computed by use of the logit model is a marginal value of time computed by means of a trade-off concept. Take the derivative of 26 with respect to $\mathrm{T}_{1}$ and $\mathrm{C}_{1},{ }^{5}$ 


$$
\frac{d(Y)}{d_{1}}=\frac{\gamma_{1}}{T_{1}} \text { and } \frac{d(Y)}{d_{1}}=\frac{\gamma_{2}}{C_{1}} \text {. }
$$

The trade off between time and cost becomes ${ }^{6}$

$$
\mathrm{T}_{\mathrm{TC}}=\frac{\frac{\mathrm{dY}}{\mathrm{dT}}}{\frac{\mathrm{dY}}{\mathrm{d} \mathrm{C}_{1}}}=\frac{\gamma_{1}}{\gamma_{2}} \cdot \frac{\mathrm{C}_{1}}{\mathrm{~T}_{1}}=\frac{\text { cents }}{\text { minutes }}
$$

This method gives the value of time spent in travel. ${ }^{7}$

The computation of time was done on thirty city pairs. Two city pairs were omitted since the probability of rail travel was extremely large. The logit expression used was

$$
\frac{p_{1}}{p_{3}}=e^{\alpha} 13\left(\frac{T_{1}}{T_{3}}\right)^{\gamma 1}\left(\frac{C_{1}}{C_{3}}\right)^{\gamma 2}
$$

where $\mathrm{p}_{1}$ is the probability of taking the rail mode, $\mathrm{p}_{3}$ is the probability of taking the bus mode, $T_{1}$ is total journey time of the rail mode (sum of access time, waiting time, mode time, and egress time), $T_{2}$ is total journey time of the bus mode (sum of access time, waiting time, mode time, and egress time), $C_{1}$ is the cost of rail mode travel, and $C_{2}$ is the cost of bus mode travel. For our particular mode the estimated logit using weighted least squares is

$$
\log \frac{\mathrm{p}_{1}}{\mathrm{p}_{3}}=\underset{(1.96)}{0.7118}-\underset{(2.59)}{1.841} \quad \log \frac{\mathrm{T}_{1}}{\mathrm{~T}_{3}}-\underset{(1.79)}{2.893} \quad \log \frac{\mathrm{C}_{1}}{\mathrm{C}_{3}}
$$

The parentheses under the regression coefficients are t-ratios. In the above expression the coefficients were significant at the five per cent level. The one detracting feature is that the correlation coefficient was . $66(\mathrm{R}=.66)$. As an alternative, the logit function 29 was also estimated for the probability ratio of air to bus. In that case the Durbin-Watson statistic was in conclusion at the five per cent level and the correlation coefficient was .83 $(R=.83)$; however, the intercept of the logit regressionwas insignificant at the five per cent level.

The above probability function for railroad and bus specifies the probability of choosing the rail mode relative to the bus. The estimates logit regression 30 corresponds to the probability of railtravel relative to bus travel in terms of their relative time and costs. In interpreting the logit regression we see that the signs are correct. The lower the bus costs relative to rail, the larger is the bus share relative to the rail share; and the lower the total journey time of bus relative to that of the rail mode, the larger is the bus share relative to the rail share of total travel. In addition, relative costs exert more of an influence on mode choice than the relative journey times of the modes.

The computed value of time from the logit expression 30 by using 28 was 2.224 cents per minute. In converting to hours the value of time spent in travel is $\$ 1.33$ per hour. The value of time was computed as atrade-off between time spent in travel and the out of the pocket costs of undertaking this journey. Thus the computed value of time reflects the ratio of compensating change in the value of one travel attribute relative to another. Again it will be pointed out that this value of time is a value without regard to mode and thus cannot be compared with studies which compute travel time by mode. 
In the work of Gary Becker[1, p. 510] the value of traveltime was stated to be approximately forty per cent of the average hourly wage rate. The Becker study would be comparable to the one undertaken here. Yet, no comparison of values would be made since Becker never stated his value of time. In an effort to see if the value of $\$ 1.33$ per hour was realistic, an average wage rate was computed for the purpose of comparison. The city pair data used in the study was obtained by aggregating eighteen Metropolitan Statistical Areas (SMSAs) intoten cities which were formed into thirty-two origin and destination pairs. In the logit model thirty city pairs were used but they involved the use of all ten cities (eighteen SMSAs). The average wage per hour was constructed for each SMSA. The average wage per hour was then weighted by the population of the SMSA, and an average wage per hour of $\$ 3.22$ was obtained for the eighteen SMSAs. At forty percent of the wage rate of value placed on time would be $\$ 1.29$ per hour. The difference between the logit value and the standard of comparison is $\$ 0.04$. Two deficiences in this comparison should be pointed out to the reader. The average wage rate standard was computed for the population of the origin destination cities and is not necessarily the average wage rate of the travelers. Secondly, the wage rate computed was done on 1960 data, while the time and cost values used in the logit estimation are for 1961. In addition to the Becker study, there are other studies which are comparable in terms of the time computation; however, none ever gave an absolute value for time. Stopher [15, p. 59] and Beesley [2, p. 184] both found that the implied value of time was forty-two per cent of the wage rate. In a computer study of urban mass transit, Charles Lave [9, p. 323] obtained a time value which was forty-two percent of the compute $r$ wage rate. In his study of the airline travel, Reuben Gronau [ 4, p. 393] also found support for the Becker results. It appears that the value of time which was computed in this study at approximately forty-one per cent of the wage rate is similar to that found in the above mentioned studies. It is hoped that this effort has provided a new dimension for mode choice models by extending them to choice decisions which areother than binary. The forty per cent rule of Becker has remained undisturbed. 


\section{FOOTNOTES}

${ }^{1}$ For use of the probit technique see Tobin [18], Kraft and Kraft [7], and Lave [9] .

${ }^{2}$ Several studies have shown the inter-urban mode choice decision is independent of income where the modes are considered to be competitive See Fisher [3]; Oi and Shuldiner [12].

${ }^{3}$ If expression 15 represented atransportation model the re would be $\mathrm{N}$ transport modes each with $n$ characteristics.

${ }^{4}$ Waiting time and the other travel time characteristics have been summed to give one time characteristic. Thus we have one time and one cost characteristic for each mode.

${ }^{5}$ Using expression 26 one could only take the derivative with respect to mode 1 or mode 3 since mode 2 is excluded from the expression. The derivative with respect to mode 3 would complicate the results of the derivation of the logit transform.

${ }^{6}$ Plourde [13, p. 41] .

${ }^{7}$ To compute the value of time of travelers, $\gamma_{1}$ and $\gamma_{2}$ are estimated by the logit regression 26 . The values of $\mathrm{C}_{1}$ and $\mathrm{T}_{1}$ are the mean values of time Plourde [13] and by Omer Saaticioglu [19]. 


\section{REFERENCES}

1. Becker, Gary S. "A Theory of the Allocation of Time. 'Economic Journal, 13 (September, 1965), 494-517.

2. Beesley, Michael E. "The Value of Time Spent in Traveling: Some New Evidence." Economica, 32 (May, 1965), 174-185.

3. Fisher, Franklin M. A Priori Information and Time Series Analysis: Essays in Economic Theory and Measurement. Amsterdam: North-Holland Publishing Co., 1962.

4. Gronau, Reuben L. "The Effect of Traveling Time on the Demand for Passenger Transportation. "Journal of Political Economy, 78 (March-April, 1970), 377-394.

5. Gronau, Reuben L. "The Effect of Traveling Time on the Demand for Passenger Airline Transportation, "Unpublished Ph. D. dissertation, Columbia University, 1967.

6. Kraft, John "Nonlinear Demand Equations for Commercial Passenger Transport Modes." Unpublished Ph. D. dissertation, University of Pittsburgh, 1971.

7. Kraft, John and Kraft, Arthur "Application of Probability Models to Evaluating Product Characteristics, " Mimeograph 1971.

8. Kraft, John and Kraft, Arthur "The Use of Linear Logit Models in Business Decisions." Mimeograph 1971.

9. Lave, Charles A. "The Demand for Urban Mass Transportation. "Review of Economics and Statistics, 52 (August, 1970), 320-323.

10. Lisco, ThomasE. "The Value of Commuter's Time: A Study in Urban Transportation, "Unpublished Ph. D. dissertation, University of Chicago, 1967.

11. Moses, Leon N. and Williamson, Harold F., Jr., "Value of Time, Choice of Mode, and the Subsidy Issue in Urban Transportation. " Journal of Political Economy, 71 (July, 1963), 247-264.

12. Oi, W. Y. and Shuldiner, D. W. An Analys is of Urban Travel Demand. Transportation Center, Evanston, Illinois: Northwestern University Press, 1962.

13. Plourde, Rodney Consumer Preferences and the Abstract Mode Model. Research Report R 68-51. Cambridge, Mass.: Department of Civil Engineering, M. I. T., 1968.

14. Saaticioglu, Omer "Toward Optimum Road Capacity and Transmit System." Unpublished Ph. D. dissertation, University of Pittsburgh, 1970.

15. Stopher, Peter R. "A Probability Model of Travel Model Choice for the Work Journey, "Highway Research Record, 282-65.

16. Theil, Henri Economics and Information Theory. Armsterdam: NorthHolland Publishing Co., 1967. 
17. Theil, Henri "A Multinomial Extension of the Linear Logit Model," International Economic Review, 10 (October, 1969), 251-259.

18. Tobin, James "The Application of Multivariate Probit Analysis to Economic Survey Data," Cowles Foundation Discussion Paper No. 1, 1955 (Unpublished). 